\title{
Cell death, proliferation and repair in human myocarditis responding to immunosuppressive therapy
}

\author{
Andrea Frustaci ${ }^{1,5}$, Cristina Chimenti ${ }^{1,5}$, Maurizio Pieroni ${ }^{2}$, Luisa Salvatori ${ }^{3}$, \\ Emanuela Morgante $^{3}$, Patrizio Sale ${ }^{3,4}$, Elisabetta Ferretti ${ }^{3}$, Elisa Petrangeli ${ }^{3,4}$, \\ Alberto Gulino ${ }^{3}$ and Matteo A Russo ${ }^{3,4}$ \\ ${ }^{1}$ 'Cuore e Grossi Vasi, Attilio Reale’ Department, La Sapienza University, Rome, Italy; ${ }^{2}$ IRCCS Ospedale \\ Multimedica, Milan, Italy; ${ }^{3}$ Experimental Medicine and Pathology Department, La Sapienza University, \\ Rome, Italy; ${ }^{4}$ IRCCS San Raffaele Pisana, Rome, Italy and ${ }^{5}$ IRCCS National Institute for Infectious \\ Diseases, Lazzaro Spallanzani, Rome, Italy
}

\begin{abstract}
In this study, we evaluate cell death, proliferation and repair in left ventricular endomyocardial biopsies from 20 patients with active lymphocytic myocarditis worsening or recovering from cardiac dysfunction after 6-months immunosuppression. Apoptosis and necrosis were assessed by in situ ligation of hairpin probes, proliferation by Ki67 and MCM5 labelling of myocytes, repair by electron microscopy, morphometric study of percent myofibrillar area and real-time polymerase chain reaction of $\alpha$-and $\beta$-Myosin Heavy Chain (MHC). Apoptosis and necrosis decreased in post- vs pretreatment biopsies by 85 and $62 \%$, respectively in responders, while increased by 42 and $46 \%$ in nonresponders. Ki67 and MCM5-positive myocytes were higher vs controls at baseline and increased by 43 and $38 \%$ at follow-up in responders and by 75 and $63 \%$ in nonresponders. Myofibrillar area reduced in pretreatment samples, increased by $33 \%$ at follow-up in responders, correlated with percent enhancement of ejection fraction and was associated with increased $\alpha$-MHC expression and $\alpha / \beta$-MHC ratio. In follow-up biopsies of nonresponders, myofibrillar area diminished by $36 \%$ and correlated with percent decrease of ejection fraction. Our results suggest that recovery of cardiac function in myocarditis responding to immunosuppression is associated with inhibition of cell death, activation of cell proliferation and with newly synthesized contractile material.
\end{abstract}

Modern Pathology (2006) 19, 755-765. doi:10.1038/modpathol.3800594; published online 31 March 2006

Keywords: myocarditis; heart failure; immunosuppressive therapy; apoptosis; myofibrillolysis; cell repair

It has been recently shown that immunosuppression can be an effective therapeutic option in active lymphocytic myocarditis, ${ }^{1,2}$ characterized by the presence of circulating serum cardiac autoantibodies and undetectable viral agents at the PCR evaluation of frozen endomyocardial samples. Cell mechanisms of cardiac recovery are, however, still speculative and may include halting of proteolysis and cell death, activation of cell proliferation and reconstitution of cell myofibrillar content. The contribution of each of these mechanisms has not yet been analyzed, while its definition may identify new strategies for the treatment of heart failure.

Correspondence: Dr A Frustaci, MD, 'Cuore e Grossi Vasi, Attilio Reale' Department, La Sapienza University, Viale Regina Elena, 324-00161 Rome, Italy.

E-mail: biocard@rm.unicatt.it

Received 29 December 2005; revised and accepted 27 February 2006; published online 31 March 2006
The aim of this study is a morphologic, morphometric and molecular evaluation of cell death, cell proliferation and repair in sequential endomyocardial biopsies of patients with myocarditis and heart failure, deteriorating and recovering, respectively, after immunosuppressive therapy.

\section{Materials and methods}

\section{Patient Selection}

Among 41 patients that at our Institution from January 1997 to July 2000 were treated for 6 months with immunosuppressive therapy (prednisone $1 \mathrm{mg}$ $\mathrm{kg}^{-1}$ day $^{-1}$ for 4 weeks followed by $0.33 \mathrm{mg} \mathrm{kg}^{-1}$ day $^{-1}$ for 5 months and azathioprine $2 \mathrm{mg} \mathrm{kg}^{-1}$ day $^{-1}$ for 6 months) in addition to full conventional therapy with digitalis, diuretics, ACE inhibitors and carvedilol because of active lymphocytic 
myocarditis and chronic heart failure, 21 responded with prompt improvement of left ventricular contractile function and reduction of left ventricular dimensions (responders) while 20 patients failed to respond and showed a persistence or a worsening of heart failure (nonresponders). ${ }^{2}$ In all, 10 responders, all showing the presence of circulating cardiac autoantibodies and absence of viral genomes in the myocardium at PCR analysis, and 10 nonresponders, characterized by worsening of left ventricular dysfunction, absence of circulating cardiac autoantibodies, and by the presence of myocardial viral genomes were retrospectively studied in order to analyze the cellular events associated with an opposite clinical outcome. The 20 patients were selected from the 41 of the original study ${ }^{2}$ on the basis of the remaining amount of myocardial material available.

\section{Clinical Studies}

Clinical assessment, resting ECG, Holter monitoring, and echocardiography were performed at baseline, weekly during the first month, every 4 weeks for the remaining 5 months. Cardiac catheterization, angiography, and biventricular endomyocardial biopsy were performed at baseline and at 6 months. Coronary angiography was performed only at baseline. All invasive cardiac procedures were performed after informed patient consent and approval by the Ethics Committees of our institution. Endomyocardial biopsies were performed in the septal-apical region of both ventricles (four to five for each ventricular chamber), as previously described. ${ }^{2}$

\section{Histologic and Immunohistochemical Studies}

Four to six endomyocardial samples obtained from each patient were processed for histological and immunohistochemical studies. Multiple 5- $\mu$ m-thick sections were stained with hematoxylin-eosin, Miller's elastic Van Gieson, Masson's trichrome and examined by light microscopy. Morphometric studies, including the assessment of the extent of myocardial fibrosis and the measurement of myocyte diameter at the level of the nucleus with computation of the cross sectional area, were performed on baseline and 6 month follow-up biopsy. ${ }^{3}$ The Dallas criteria ${ }^{4}$ were adopted for histological diagnosis of myocarditis, supplemented by immunohistochemistry for the characterization of inflammatory infiltrates. ${ }^{5}$

\section{Transmission Electron Microscopy and Morphometry Studies}

Ultrathin sections were stained with uranyl acetate and lead hydroxide. Morphometric analysis of myofibrillar components was performed on baseline and
6 month follow-up left ventricular biopsy specimens from all patients and was carried out by a computer assisted image analyzer, utilizing the KS-300 software (Carl Zeiss Co, Germany, 1995), directly on photographic negatives of TEM sections. The overall procedure and measurements have been described in detail in a previous paper. ${ }^{6}$ In all, 20 surgical specimens of papillary muscles from age and sexmatched patients with mitral stenosis and normal left ventricular function were used as normal controls.

\section{Molecular Biology Studies}

Two frozen myocardial specimens from each patient were used for PCR and reverse transcriptase PCR analysis to detect cardiotropic DNA (Adenovirus, Epstein Barr virus, Herpes Simplex viruses, Cytomegalovirus, Parvovirus B19) and RNA viruses (Enterovirus, Influenza viruses A and B, Hepatitis $C$ virus), using the primers previously described ${ }^{2-5}$ in baseline and follow-up biopsies. Total RNA extracted from additional left ventricular specimens of responders patients at baseline and 6 month follow-up were used for real-time PCR, using the ABI Prism 7700 Sequence Detection System (Applied Biosystems, Foster City, CA, USA), to detect myosin heavy chain (MHC) $\alpha$ and MHC $\beta$. For each gene amplification a standard curve was generated using serial dilutions of human heart total RNA (BD Biosciences Clontech, Palo Alto, CA, USA). Quantification results were showed as MHC $\alpha$ and $\beta$ mRNA expression normalized with the endogenous control glyceraldehyde-3-phosphate dehydrogenase. ${ }^{7}$ Frozen ventricular myocardial samples obtained during cardiac surgery from 20 age-matched patients with mitral stenosis and normal left ventricular function served as normal controls.

\section{Evaluation of Cell Apoptosis, Necrosis, and Proliferation}

In situ ligation of hairpin probes with single-base $3^{\prime}$ overhangs (hairpin 1) or blunt ends (hairpin 2) were used to measure, respectively, apoptosis and necrosis of myocytes ${ }^{3,8,9}$ on baseline and follow-up left ventricular endomyocardial biopsies. The number of proliferating myocytes was evaluated by Ki67 (clone MIB-1, Diagnostic Biosystems, Pleasanton, CA, USA), and MCM5 (Accurate Chemicals, Westbury, NY, USA), two nuclear proteins involved in the cell cycle. ${ }^{10-12}$ Myocytes were labeled by $\alpha$-sarcomeric actin antibody staining (clone C5C, Sigma, St Louis, MO, USA). Nuclei were stained with propidium iodide. Specimens were examined with confocal microscope. ${ }^{3}$ Control values for myocyte death and proliferation were assessed in 20 surgical specimens of papillary muscles from patients with mitral stenosis and normal left ventricular function. 


\section{Statistical Analysis}

Data are presented as mean \pm s.d. A value of $P<0.05$ was considered as significant. Difference between two groups was determined by unpaired $t$-test for continuous variables and Fisher's exact test for categorical data. Changes observed before and after immunosuppressive treatment were examined by paired $t$-test. The $F$ test was used to compare variances. The bivariate correlation between ejection fraction and myofibrillar area was explored with Pearson correlation.

\section{Results}

Comparison of baseline clinical, morphometric, and histochemical parameters between responders and nonresponders are presented in Table 1.
In responders cardiac function improved significantly after 6-month immunosuppression, while it failed to improve or deteriorated in nonresponders (Tables 1 and 2, Figures 1a and 2a).

\section{Immunohistological Studies}

Histological and immunohistochemical analysis showed, in all patients, an active lymphocytic myocarditis with diffuse inflammatory infiltrates associated with focal necrosis and diffuse vacuolar degeneration of the adjacent myocytes (Figure 1b, left panel, Figure 2b left panel) with interstitial and focal replacement fibrosis. The infiltrates included mainly activated $\mathrm{T}$ lymphocytes $(\mathrm{CD} 45 \mathrm{RO}+$ ) with a moderate amount of cytotoxic lymphocytes $(\mathrm{CD} 8+)$. Follow-up histology at 6 months showed in responders replacement of myocardiocytes

Table 1 Data on 20 patients with active lymphocytic myocarditis responding $(n=10)$ and nonresponding ( $n=10)$ to immunosuppression

\begin{tabular}{|c|c|c|c|}
\hline & Responders $(\mathrm{n}=10)$ & Non responders $(\mathrm{n}=10)$ & $\mathrm{P}$-value \\
\hline Age (years) & $38.6 \pm 14.3$ & $45.5 \pm 12.9$ & NS \\
\hline Sex (female/male) & $5 / 5$ & $3 / 7$ & NS \\
\hline Months since disease onset & $9.7 \pm 1.6$ & $10.1 \pm 1$ & NS \\
\hline NYHA class (III/IV) & $1 / 7$ & $5 / 5$ & NS \\
\hline LVEDD (mm) & $64.1 \pm 8.5$ & $65.5 \pm 4.3$ & NS \\
\hline LVEDV (ml) & $206.3 \pm 53.1$ & $198.3 \pm 29.1$ & NS \\
\hline $\operatorname{LVEF}(\%)$ & $23.6 \pm 3.2$ & $29.2 \pm 3.2$ & 0.001 \\
\hline LVEDP (mmHg) & $22.4 \pm 8.4$ & $21.4 \pm 8.3$ & NS \\
\hline PASP (mmHg) & $38.3 \pm 13.5$ & $40.0 \pm 14$ & NS \\
\hline Cardiac index $\left(\mathrm{l} \mathrm{min}^{-1} \mathrm{~m}^{-2}\right)$ & $1.8 \pm 0.4$ & $1.9 \pm 0.2$ & NS \\
\hline Myofibrillar area (\%) & $40.61 \pm 2$ & $39.21 \pm 3.7$ & NS \\
\hline Myocardial fibrosis (\%area) & $8 \pm 3.4$ & $7.5 \pm 2.9$ & NS \\
\hline Myocyte trasverse area $\left(\mu \mathrm{m}^{2}\right)$ & $414 \pm 158$ & $442 \pm 104$ & NS \\
\hline Apoptosis (myocyte/106) & $2285 \pm 764$ & $2134 \pm 765$ & NS \\
\hline Necrosis (myocyte/106 & $16151 \pm 6082$ & $15346 \pm 3048$ & NS \\
\hline Ki-67 (myocyte/10 ${ }^{6}$ ) & $20044 \pm 3465$ & $19682 \pm 4241$ & NS \\
\hline MCM5 (myocyte/106) & $34393 \pm 7324$ & $36377 \pm 5260$ & NS \\
\hline
\end{tabular}

Continuous variables are expressed as mean \pm s.d. NYHA indicates New York Heart Association, LV, left ventricle; EDD, end-diastolic diameter; EDV, end-diastolic volume; EF, ejection fraction; EDP, end-diastolic pressure; PASP, pulmonary artery systolic pressure.

Table 2 Clinical, echocardiographic and ultrastructural data on the 10 patients with myocarditis before and after 6 months of successful immunosuppressive therapy

\begin{tabular}{|c|c|c|c|c|c|c|c|c|c|c|c|}
\hline \multirow[t]{2}{*}{ Patient } & \multirow[t]{2}{*}{ Age (years) } & \multirow[t]{2}{*}{ Sex } & \multirow[t]{2}{*}{ Viral agent } & \multicolumn{4}{|c|}{ Baseline } & \multicolumn{4}{|c|}{ After 6-month IT } \\
\hline & & & & $\begin{array}{c}\text { NYHA } \\
\text { class }\end{array}$ & $\begin{array}{c}E D D \\
(m m)\end{array}$ & $E F(\%)$ & $\begin{array}{c}\text { Myofibrillar } \\
\text { area }(\%)\end{array}$ & $\begin{array}{c}\text { NYHA } \\
\text { class }\end{array}$ & $\begin{array}{l}E D D \\
(m m)\end{array}$ & $E F(\%)$ & $\begin{array}{c}\text { Myofibrillar } \\
\text { area }(\%)\end{array}$ \\
\hline 1 & 35 & $\mathrm{M}$ & No & IV & 68 & 24 & 39.7 & I & 55 & $51 *$ & $52.7^{*}$ \\
\hline 2 & 37 & $\mathrm{M}$ & No & IV & 62 & 23 & 42.2 & I & 56 & $54^{*}$ & $58.8^{*}$ \\
\hline 3 & 33 & $\mathrm{~F}$ & No & IV & 59 & 29 & 43.1 & I & 57 & $47^{*}$ & $50.3^{*}$ \\
\hline 4 & 59 & $\mathrm{~F}$ & No & III & 46 & 23 & 39.3 & I & 47 & $48^{*}$ & $53.1^{*}$ \\
\hline 5 & 50 & $\mathrm{M}$ & No & IV & 71 & 26 & 42.7 & II & 61 & $45^{*}$ & $51.3^{*}$ \\
\hline 6 & 15 & $\mathrm{M}$ & No & IV & 75 & 27 & 41.3 & I & 67 & $44^{*}$ & $51.6^{*}$ \\
\hline 7 & 54 & $\mathrm{~F}$ & No & IV & 62 & 21 & 39.3 & I & 52 & $53^{*}$ & $58.3^{*}$ \\
\hline 8 & 23 & $\mathrm{~F}$ & No & IV & 61 & 18 & 37.3 & I & 54 & $49^{*}$ & $58.2^{*}$ \\
\hline 9 & 30 & $\mathrm{~F}$ & No & IV & 63 & 21 & 38.7 & I & 49 & $40^{*}$ & $50.1^{*}$ \\
\hline 10 & 50 & $\mathrm{M}$ & No & IV & 74 & 24 & 42.5 & I & 58 & $47^{*}$ & $54.4^{*}$ \\
\hline
\end{tabular}

IT, immunosuppressive therapy; NYHA indicates New York Heart Association; EDD, end-diastolic diameter; EF, ejection fraction.

${ }^{*} P>0.001$ vs baseline values. 

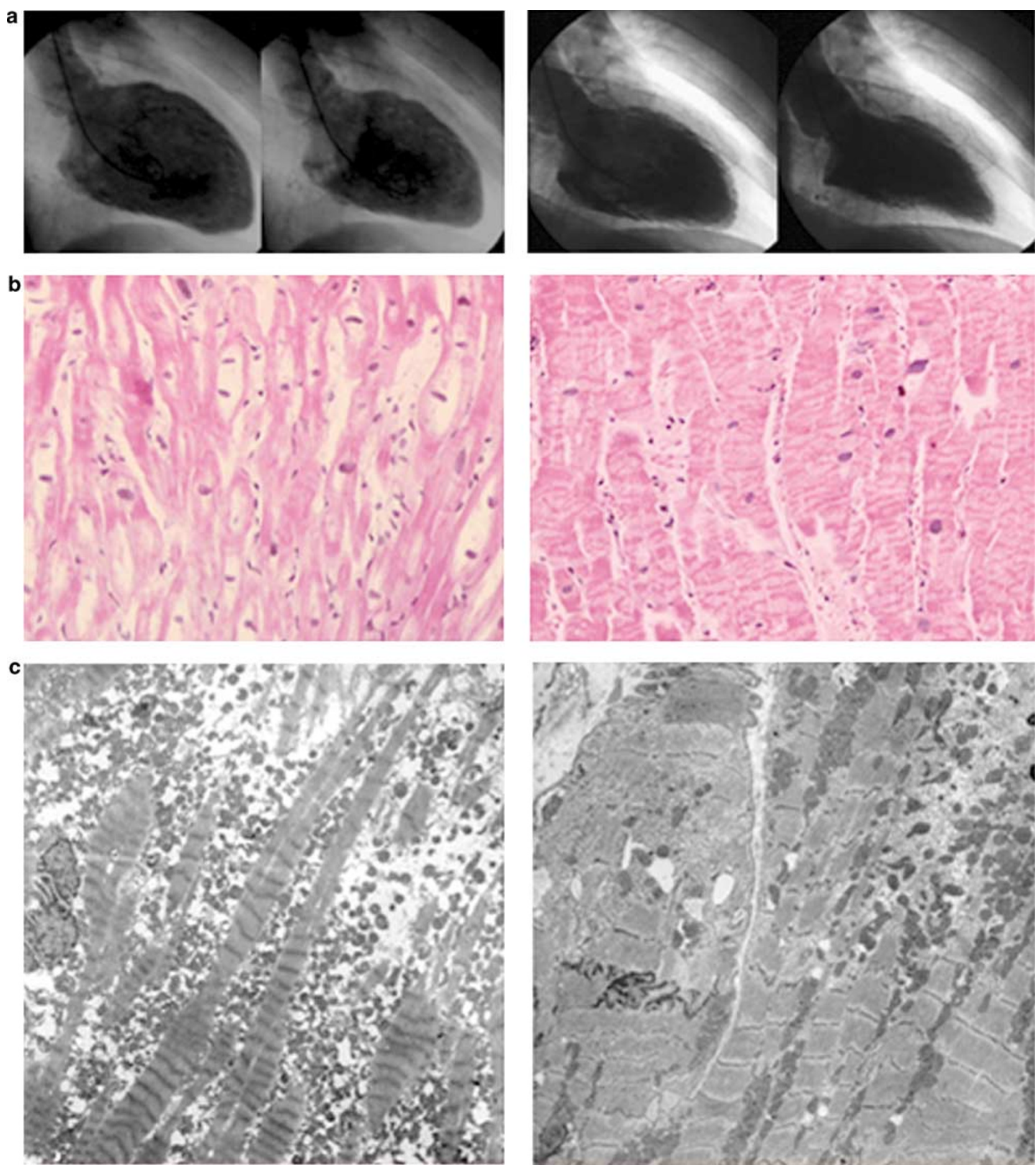

Figure 1 (a) Left ventricular angiography of a responder patient (no. 7) before (left panel) and after 6 months of immunosuppressive treatment (right panel), showing a dilated and hypokinetic ventricle, which recovered at 6-month follow-up with reduction of left ventricular dimensions and improvement of contractility; (b) Histology from the same patient. At baseline biopsy (left panel) an active myocarditis with inflammatory infiltrates associated with focal necrosis and diffuse vacuolar degeneration of the adjacent myocytes is observed. At control biopsy (right panel) myocarditis progressed to a healed phase with filling of myocardiocytes vacuolar spaces with myofibrillar material (hematoxylin and eosin, magnification $=100 \times$ ); $(\mathbf{c})$ Transmission electron microscopy from the same patient: before treatment (left panel) areas of myofibrillolysis are evident, occupied by cytosolic components. After immunosuppressive treatment (right panel), the empty cytosolic areas disappeared and the overall ultrastructure appears to be similar to a normal myocardium (scale bar $=1 \mu \mathrm{m})$.

vacuolar spaces with myofibrillar material and the inflammatory process progressed to a healed phase (Figure 1b, right panel). Conversely, in nonrespon- ders follow-up histology revealed diffused and more pronounced myocyte degeneration with disappearance of inflammatory infiltrates (Figure 2b, right 

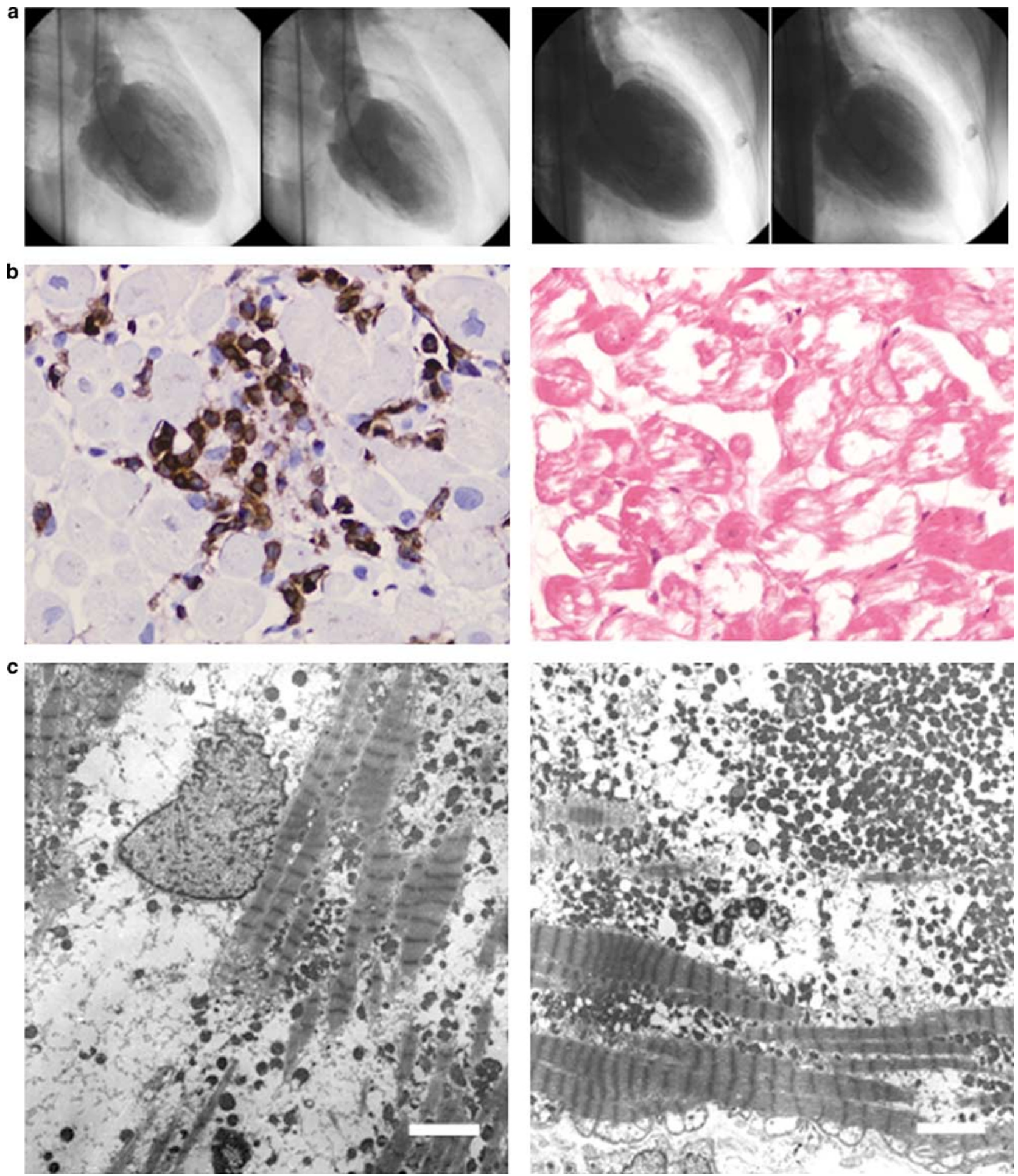

Figure 2 (a) Left ventricular angiography of a nonresponder patient (no. 6) before (left panel) and after 6 months of immunosuppressive treatment (right panel) showing a dilated and hypokinetic ventricle, with a further dilation and impaired contractility at 6-month followup; (b) Histology from the same patient. At baseline biopsy (left panel) an active lymphocytic myocarditis with inflammatory infiltrates represented by activated $\mathrm{T}$ lymphocytes $(\mathrm{CD} 45 \mathrm{RO}+$ ) (immunoperoxidase, magnification $=250 \times$ ), is evident. At control biopsy (right panel) myocarditis evolved to a dilated cardiomyopathy with myocyte degeneration and disappearance of lymphocytic infiltrates (hematoxylin and eosin $250 \times$ ); (c) Transmission electron microscopy from the same patient: before treatment (left panel) areas of myofibrillolysis are evident. These areas were more pronounced after 6 month immunosuppressive treatment (right panel) (scale bar $=1 \mu \mathrm{m})$.

panel). No evidence of myocardial inflammation was observed in normal controls' biopsies. Myocardial fibrosis and myocyte transverse area increased in both responders and not responders at baseline compared with controls $(P<0.001)$ (fibrosis: controls $=4.5 \pm 2.0 \%$, responders $=8.0 \pm 3.4 \%$, not 
responders $=7.5 \pm 2.9 \%$; myocyte area: controls $=$ $295 \pm 45 \mu \mathrm{m}^{2}$, responders $=414 \pm 158 \mu \mathrm{m}^{2}$, not responders $=441 \pm 103 \mu \mathrm{m}^{2}$ ). Fibrosis further increased by $50 \%$ (pretreatment $=8.0 \pm 3.4 \%$, post-treatment $=$ $12.0 \pm 3.5 \%, P<0.001)$ in responders and by $140 \%$ (pretreatment $=7.5 \pm 2.9 \%$, post-treatment $=18.0 \pm$ $5.3 \%$ ) in nonresponders. In comparison with pretreatment samples, the transverse area of myocytes was $45 \%$ bigger (pretreatment $=414 \pm 158 \mu \mathrm{m}^{2}$, posttreatment $\left.=601 \pm 153 \mu \mathrm{m}^{2}, \quad P<0.001\right)$ in follow-up biopsies of responders, while it remained unchanged (pretreatment $=441 \pm 103 \mu \mathrm{m}^{2}$, post-treatment $=479 \pm$ $111 \mu \mathrm{m}^{2}$ ) in follow-up biopsies of nonresponders.

\section{Electron Microscopy and Morphometry Studies}

Transmission electron microscopy studies were aimed to characterize at subcellular level the myocardiocyte damage and its recovery or worsening after 6 months of immunosuppressive treatment. In all patients before treatment, myofibrillolysis was present in all myocardiocytes analyzed, appearing as large cytoplasmic areas, apparently empty or filled with fine granular material and localized among thin bundles of sarcomeric fibrils (Figure 1c left panel, Figure 2c left panel). These areas were absent in myocardiocytes of normal controls.

At transmission electron microscopy and morphometric analysis, the comparison with normal myocardium showed that such empty areas were the result of the reduction of myofibrillar content. There was not statistically significant difference in terms of myofibrillar area content between responders and nonresponders at baseline biopsy (Table 1). After 6 month immunosuppressive treatment, in responders myofibrillar mass and architecture recovered and appeared to be similar to those of a normal myocardium. Large, apparently empty, cytosolic spaces disappeared, being replaced by regularly organized sarcomeres and normal mitochondria (Figure 1c, right panel). This was confirmed by morphometry which showed that the percentage of myofibrillar area on total cellular area was increased by $33 \%$ proportionally to the recovery of left ventricular ejection fraction (Pearson correlation 0.84, $P<0.01$ ) (Table 2 and Figure 3a). Conversely, in myocytes of not responders there was a further reduction of myofibrillar content (Figure 2c, right panel), corresponding to a $36 \%$ reduction of percentage of myofibrillar area at morphometric analysis (Table 3 and Figure $3 \mathrm{~b}$ ) paralleled by a decrease in left ventricular ejection fraction (Pearson correlation $0.72, P<0.01)$.

\section{Molecular Biology Studies}

The presence of sufficient target nucleic acid for PCR analysis was confirmed by amplification of ${ }^{\circledR}$-globin for DNA and glyceraldehyde-3-phosphate dehydrogenase for RNA in all cases in baseline and
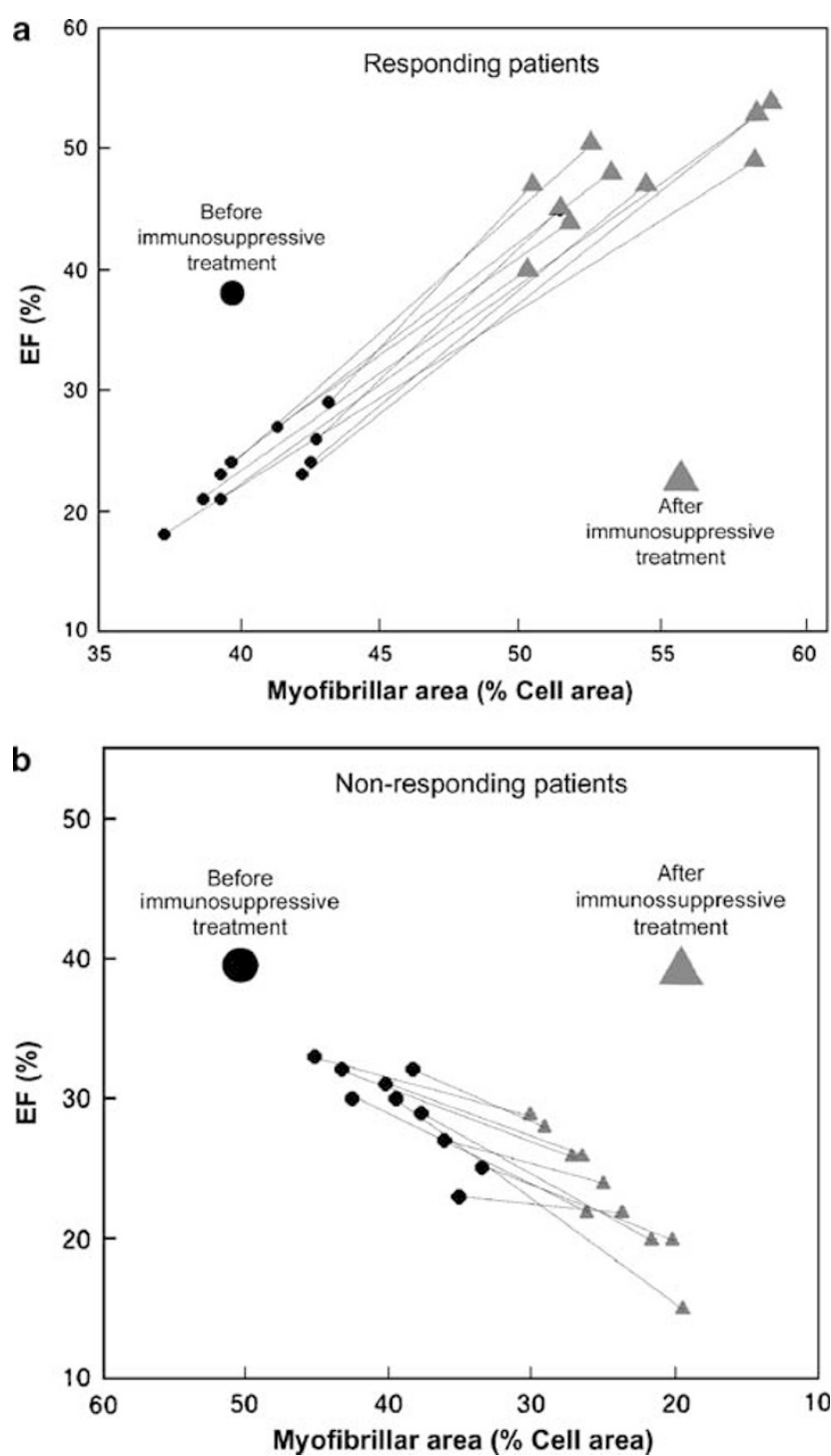

Figure 3 Correlation between myofibrillar area and LV ejection fraction before after immunosuppressive treatment in each responding (a) or nonresponding patient (b). Statistical evaluation: (a): $R=0.84, P<0.001$. (b): $R=0.72, P<0.001$.

follow-up biopsies. Viral genomes were absent both in baseline and follow-up biopsies of responders and in normal controls. A myocardial viral infection was detected in all nonresponders (Tables 2 and 3). Importantly, follow-up biopsies showed a persistence of the viral genome in all cases.

The relative quantitative analysis (Figure 4) showed that the expression of the MHC $\alpha$ and $\beta$ isoforms in the group of patients before treatment was 34 and $19 \%$ lower than in controls ( 0.56 vs 0.85 , and 5.2 vs 6.4, respectively). After therapy, a significant increase in the levels of MHC $\alpha$ mRNA (5.5fold, from 0.56 to $3.1, P<0.001$, comparing the variances) and a not significant trend to decrease in MHC $\beta$ expression (0.8-fold, from 5.2 to 4.2 , $P=0.28$ ) was observed, leading to a marked increase in MHC $\alpha / \beta$ ratio (6.7-fold). 
Table 3 Clinical, echocardiographic and ultrastructural data on the 10 patients with active lymphocytic myocarditis not responding to immunosuppressive therapy

\begin{tabular}{|c|c|c|c|c|c|c|c|c|c|c|c|}
\hline \multirow[t]{2}{*}{ Patient } & \multirow[t]{2}{*}{ Age (years) } & \multirow[t]{2}{*}{ Sex } & \multirow[t]{2}{*}{ Viral agent } & \multicolumn{4}{|c|}{ Baseline } & \multicolumn{4}{|c|}{ After 6-month IT } \\
\hline & & & & $\begin{array}{c}\text { NYHA } \\
\text { class }\end{array}$ & $\begin{array}{c}E D D \\
(m m)\end{array}$ & $E F(\%)$ & $\begin{array}{c}\text { Myofibrillar } \\
\text { area }(\%)\end{array}$ & $\begin{array}{c}\text { NYHA } \\
\text { class }\end{array}$ & $E D D(\mathrm{~mm})$ & $E F(\%)$ & $\begin{array}{c}\text { Myofibrillar } \\
\text { area }(\%)\end{array}$ \\
\hline 1 & 62 & $\mathrm{~F}$ & EBV & IV & 66 & 23 & 35.2 & IV & 67 & $22 *$ & $26.3^{*}$ \\
\hline 2 & 29 & $\mathrm{~F}$ & Influenza A & IV & 60 & 33 & 45.2 & IV & 62 & $29 *$ & $30.2^{*}$ \\
\hline 3 & 37 & $\mathrm{M}$ & PVB19 & III & 70 & 30 & 42.6 & III & 73 & $22^{*}$ & $23.7^{*}$ \\
\hline 4 & 37 & $\mathrm{M}$ & Enterovirus & III & 63 & 32 & 43.4 & IV & 63 & $26^{*}$ & $27.3^{*}$ \\
\hline 5 & 50 & $\mathrm{M}$ & EBV & IV & 61 & 25 & 33.6 & IV & 75 & $20^{*}$ & $20.2^{*}$ \\
\hline 6 & 59 & $\mathrm{M}$ & Adenovirus & III & 68 & 30 & 39.6 & IV & 69 & $15^{*}$ & $19.5^{*}$ \\
\hline 7 & 41 & $\mathrm{M}$ & Adenovirus & III & 73 & 31 & 40.2 & IV & 76 & $26^{*}$ & $26.6^{*}$ \\
\hline 8 & 27 & $\mathrm{M}$ & Enterovirus & IV & 69 & 29 & 37.8 & III & 77 & $20^{*}$ & $21.7^{*}$ \\
\hline 9 & 57 & $\mathrm{~F}$ & EBV & III & 55 & 32 & 38.3 & III & 60 & $28 *$ & $29.2^{*}$ \\
\hline 10 & 56 & $\mathrm{M}$ & Adenovirus & IV & 62 & 27 & 36.2 & IV & 62 & $24^{*}$ & $25.1^{*}$ \\
\hline
\end{tabular}

IT, immunosuppressive therapy; NYHA indicates New York Heart Association; EDD, end-diastolic diameter; EF, ejection fraction. ${ }^{*} P>0.001$ vs baseline values.
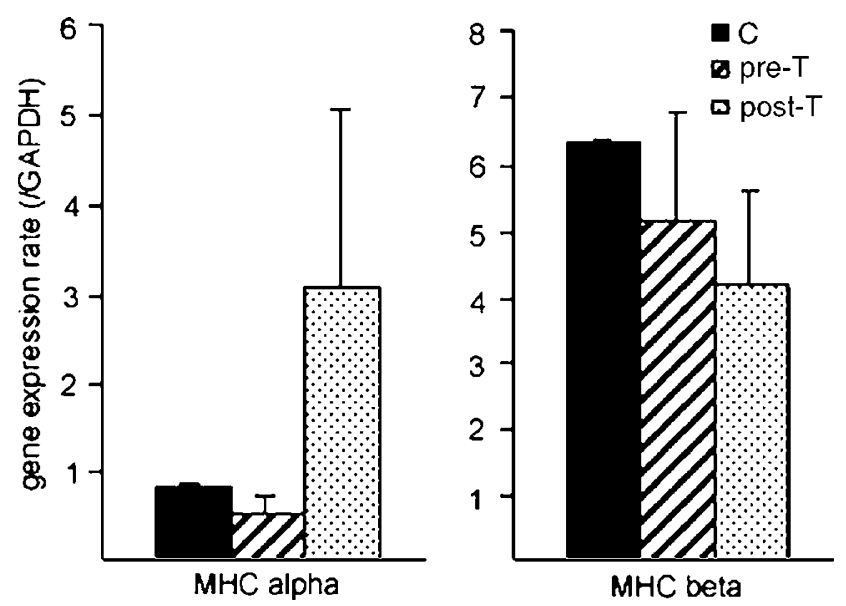

Figure 4 Gene expression rate in responders of MHC $\alpha$ and MHC $\beta$ in pretreatment (pre-T), post-treatment (post-T) and control (C) hearts. The expression ratio between each target gene and GAPDH calculated in all samples analyzed are shown as means \pm s.e.

\section{Cell apoptosis, Necrosis, and Proliferation}

Myocardiocyte apoptotic and necrotic cell death were, respectively, 104 -fold (patients $=2285 \pm 764$ / $10^{6}$, controls $=22 \pm 10 / 10^{6}, P<0.001$ ) and 61 -fold (patients $=16151 \pm 2792 / 10^{6}$, controls $=265 \pm 97 / 10^{6}$, $P<0.001)$ greater in baseline biopsies of responders than in controls (Figure 5). Importantly, after 6 months of immunosuppressive therapy, apoptosis and necrosis decreased by $85 \% \quad\left(353 \pm 93 / 10^{6}\right.$ vs $\left.2285 \pm 764 / 10^{6}, P<0.001\right)$ and $62 \%(6082 \pm 2126 \mathrm{Vs}$ $16151 \pm 2792 / 10^{6}, P<0.001$ ) respectively (Figure 5).

In nonresponders myocyte apoptosis was 97 -fold higher (patients $=2133 \pm 765 / 10^{6}$, controls $=22 \pm 10 /$ $\left.10^{6}, P<0.001\right)$ and necrosis was 58-fold higher (patients $=15345 \pm 3047 / 10^{6}$, controls $=265 \pm 97 / 10^{6}$, $P<0.001$ ) in baseline biopsy compared with controls, and further increased by $42 \%\left(3032 \pm 930 / 10^{6}\right.$ vs $\left.2133 \pm 765 / 10^{6}, P<0.001\right)$ and by $46 \%(22460 \pm 2518$ vs $\left.15345 \pm 3047 / 10^{6}, P<0.001\right)$ respectively, in follow-up biopsies.
The assessment of myocyte proliferation in baseline biopsy tissue of responders compared with controls showed 5.2-fold (patients $=20044 \pm 3465 / 10^{6}$, controls $=3817 \pm 943 / 10^{6}, P<0.001$ ) and 6.6-fold (patients $=34393 \pm 7324 / 10^{6}$, controls $=5202 \pm 1290 / 10^{6}$, $P<0.001)$ higher labeling of myocyte nuclei by Ki67 and MCM5, respectively. The level of myocyte replication was increased by $43 \%$ for Ki-67 $\left(28697 \pm 5141 / 10^{6}\right.$ vs $\left.20044 \pm 3465 / 10^{6}, \quad P<0.001\right)$ and by $38 \%$ for MCM5 (47 420 $\pm 9476 / 10^{6}$ vs 34393 $\left.\pm 7324 / 10^{6}, P<0.001\right)$ after 6 months of successful immunosuppression (Figure 6). Similarly, the number of cycling myocytes in baseline myocardial tissue of nonresponders was greater that in controls (Ki67 labeling: $\quad$ atients $=19682 \pm 4241 / 10^{6}$, controls $=$ $3817 \pm 943 / 10^{6}, P<0.001$, 5.1-fold increase; MCM5 labeling: patients $=36377 \pm 5259 / 10^{6}$, controls $=5202$ $\pm 1290 / 10^{6}, P<0.001$, seven-fold increase) and further increase by $75 \%$ (Ki67 labeling: $34487 \pm 6595 / 10^{6}$ Vs $\left.19682 \pm 4241 / 10^{6}, \quad P<0.001\right)$ and $63 \% \quad$ (MCM5 labeling: $59364 \pm 7117 / 10^{6}$ vs $36377 \pm 5259 / 10^{6}$, $P<0.001$ ) after immunosuppression (Figure 6).

\section{Discussion}

After controversial results from the systematic use of immunosuppression in the treatment of myocarditis, ${ }^{13,14}$ recent reports seem to have identified biological markers recognizing specific subset of responders. In particular, patients with active lymphocytic myocarditis and myocyte expression of HLA molecules, ${ }^{1}$ absence of myocardial viral genome at PCR and detectable cardiac autoantibodies in the serum ${ }^{2}$ have been found highly susceptible to immunosuppressive therapy with significant or complete recovery of a severely depressed cardiac contractility in comparison with virus positive and cardiac autoantibodies negative patients. However, cell mechanisms of cardiac recovery are still speculative and the impact of cell repair vs cell 


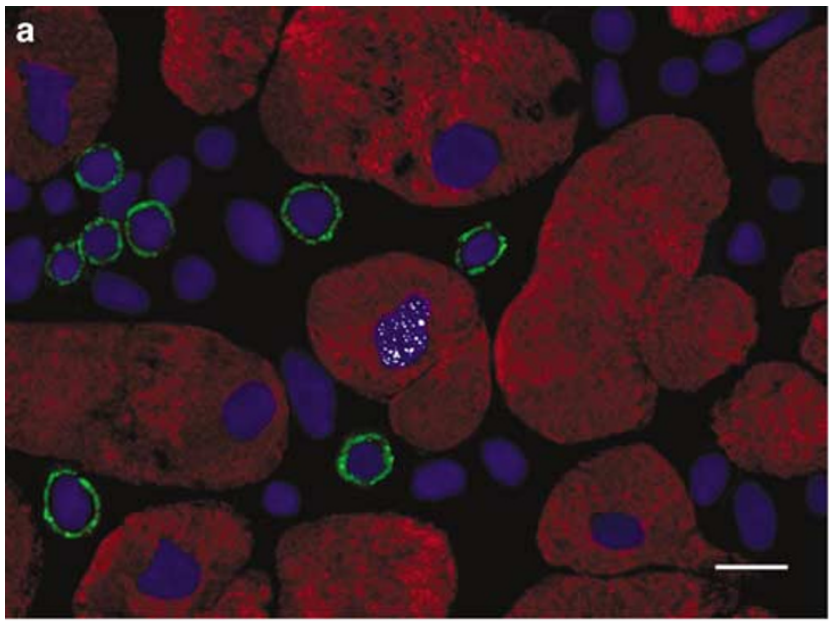

\section{b}
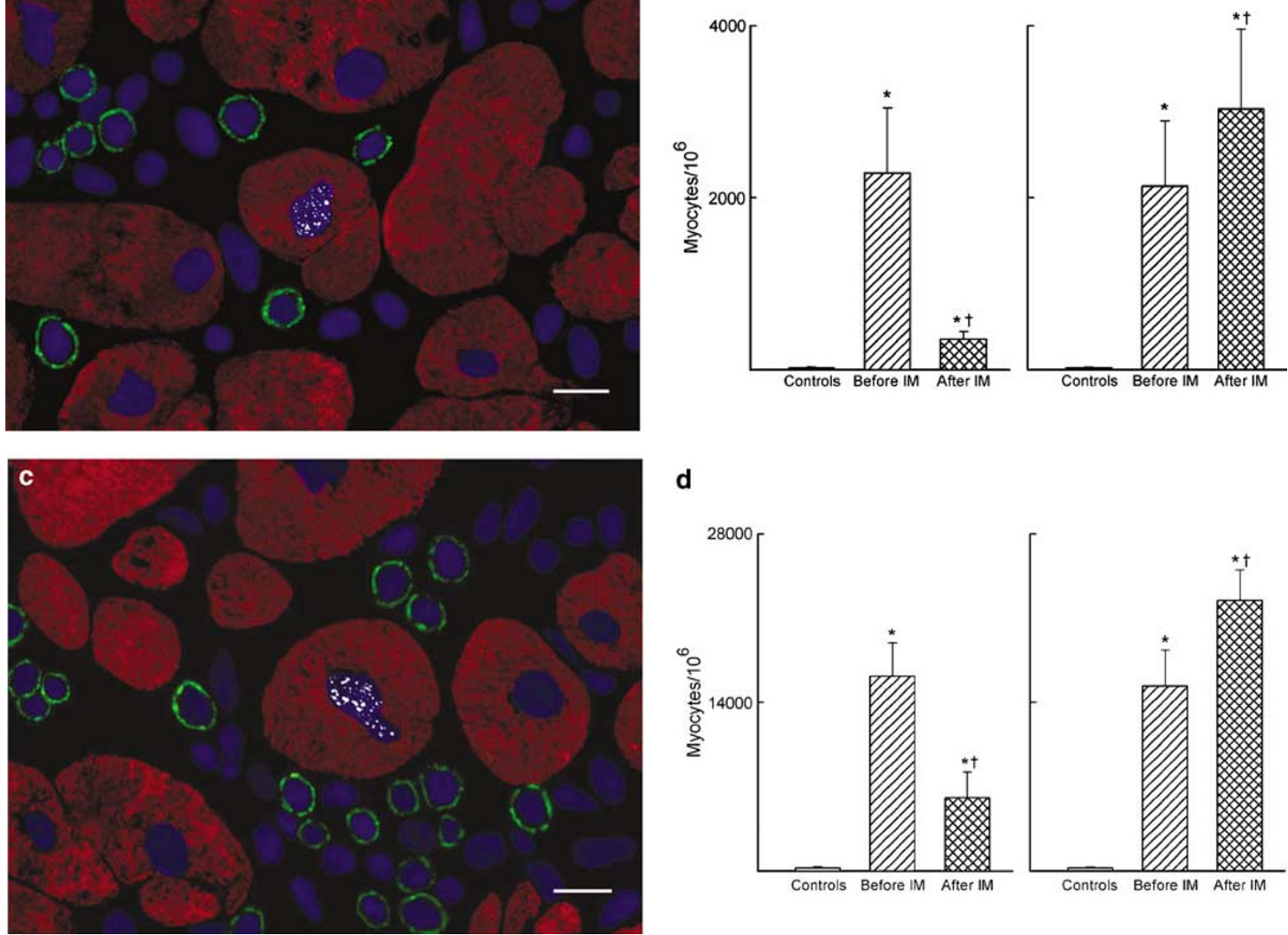

d

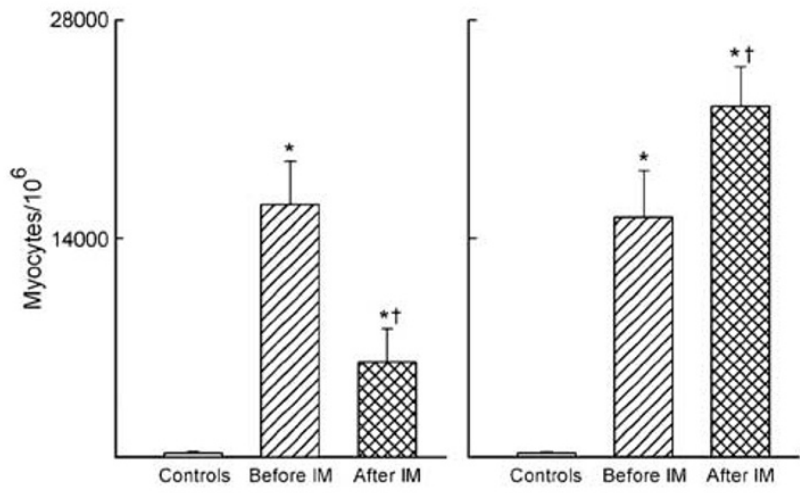

Figure 5 (a) Left ventricular endomyocardial biopsy of a responding patient, showing a myocyte apoptotic nucleus (white dots). Myocytes are identified by the red fluorescence of $\alpha$-sarcomeric actin antibody staining of myocyte cytoplasm. Lymphocytes are recognized by green fluorescence of CD45RO on the cell surface. Nuclei are stained by the blue fluorescence of propidium iodide. Scale bar $=10 \mu \mathrm{m}$. (b) Number of apoptotic myocytes in responding (left) and in nonresponding (right) patients before and after 6-month immunosuppressive therapy (IM) compared with normal controls. Results are mean \pm s.d. * and $\dagger$ indicate significant difference vs controls and baseline values, respectively. (c) Left ventricular endomyocardial biopsy of a nonresponding patient, illustrating a myocyte (red fluorescence) necrotic nucleus (white dots) surrounded by CD45RO + lymphocytes (green fluorescence). Nuclei are stained by the blue fluorescence of propidium iodide. Scale bar $=10 \mu \mathrm{m}$. (d) Number of necrotic myocytes in responding (left) and in nonresponding (right) patients before and after 6-month immunosuppressive therapy (IM) compared with normal controls. Results are mean \pm s.d. * and $\dagger$ indicate significant difference vs controls and baseline values, respectively.

proliferation or the possible contribute of cell death inhibition are still unknown.

In the present study, the authors have documented that all the aforementioned cell mechanisms intervene in the process of cardiac recovery, although with a different relevance that can be perceived or enhanced in other forms of heart failure not responding to conventional therapeutic strategies.

\section{Role of Cell Death}

In the present study, it has been shown that myocarditis manifesting with chronic heart failure, is characterized by a remarkable increase of cell death by apoptosis and necrosis. Indeed, apoptosis and necrosis were several times higher in myocarditis patients compared with normal controls and these values were comparable with those reported in severe forms of dilated cardiomyopathy, ${ }^{3,8,15}$ suggesting that myocyte loss is an important mechanism of myocardial damage in myocarditis with cardiac dysfunction. On follow-up biopsies of responders cell death decreased consistently, specifically by $68 \%$ for cell necrosis and $86 \%$ for cell apoptosis, allowing cell repair and proliferation to become effective biological events for the improvement of cardiac function. Several mechanisms may have concurred to cell rescue including: (a) removal of natural killer cells and activated T-lymphocytes that with their perforins and granzymes produce damage of myocyte membrane inducing cell necrosis and DNA fragmentation leading to apoptosis; ${ }^{16,17}$ (b) Inhibition of cytokine and free radical production (particularly TNF- $\alpha$ and IL-1) that can activate 

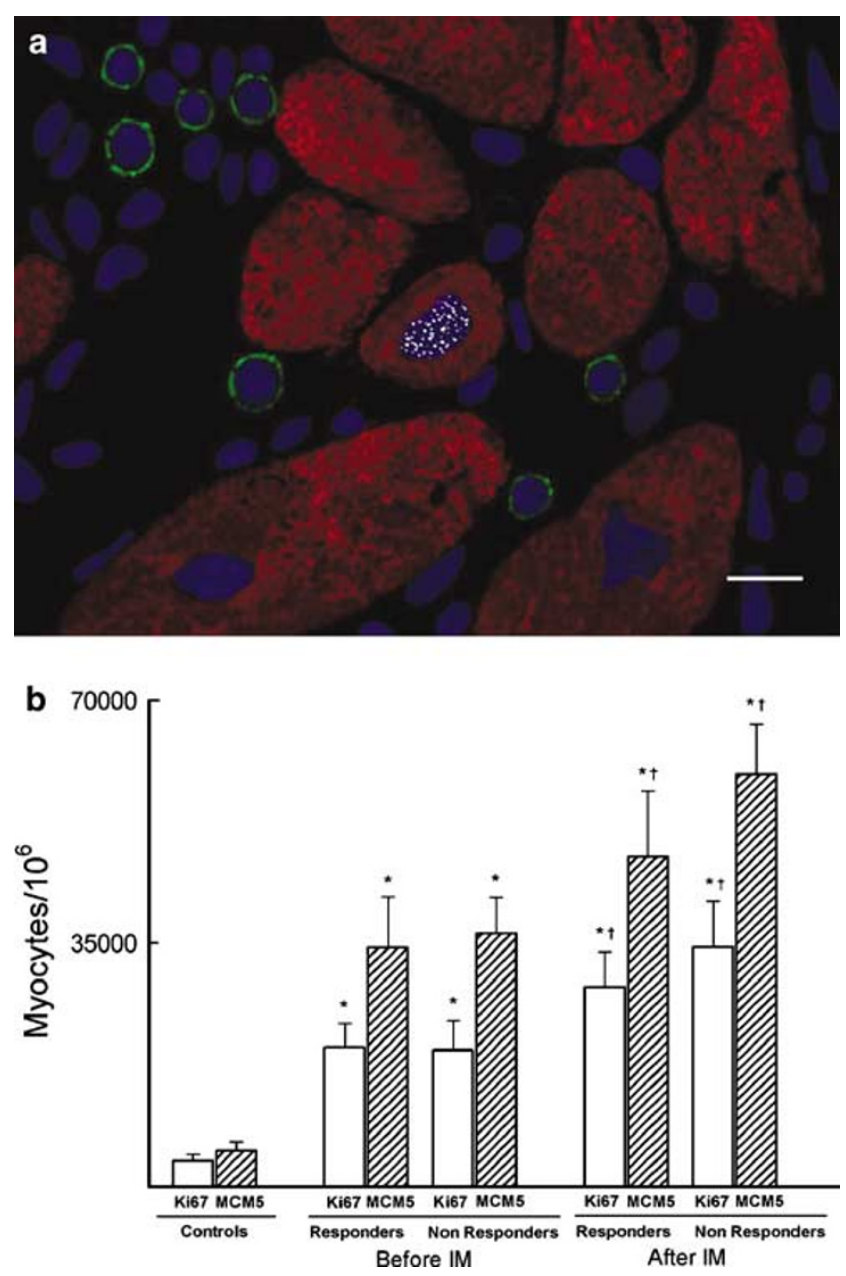

Figure 6 (a) Left ventricular endomyocardial biopsy of a responding patient showing a MCM5 positive nucleus (white dots) of a myocyte (red fluorescence, $\alpha$-sarcomeric actin). Lymphocytes are recognized by green fluorescence of CD45RO on the cell surface. Nuclei are stained by propidium iodide (blue fluorescence). Scale bar $=10 \mu \mathrm{m}$. (b) Number of Ki67 and MCM5 positive myocytes in controls, responder and nonresponder patients, before and after 6 month immunosuppression (IM). $*$ and $\dagger$ indicate a significant difference vs controls and pretreatment biopsy, respectively.

intracellular caspase cascade and ultimately myocyte apoptosis; ${ }^{18}$ (c) reduction of reactive oxygen species production (including NO), that at high levels can induce necrosis while at lower levels can trigger apoptosis; ${ }^{19}$ (d) reduction of left ventricular load due to the improvement of cardiac dimensions and contractility. ${ }^{20}$

It is noteworthy that even severe degrees of cell death can be remarkably slowed or stopped if the treatment is really able to interfere with the underlying mechanism of damage.

On the other hand, the increased level of cell death observed in follow-up biopsy of not responders correlates with the worsening of heart failure and with the persistence of viral agents, that have been shown to induce cardiomyocyte apoptosis in myocarditis. ${ }^{21}$

\section{Role of Cell Regeneration}

The presence of proliferating myocytes in the heart of patients with myocarditis was demonstrated by the nuclear expression of Ki-67 and MCM5. Ki-67 and MCM5 positive cells were significantly higher compared with normal controls in both responders and nonresponders, suggesting that in chronic myocarditis, as in other forms of heart failure, there is an activation of myocyte regeneration in the attempt to compensate cell loss. Thus, the increased expression of preadult cardiac myosin isoforms found in our patients could be partially related to the presence of immature myocytes.

The source of replicating myocytes could not be investigated because of the limited amount of myocardial material. However, according to recent studies, undifferentiated and early committed cells, expressing stem cell markers and telomerase, are present in the adult heart and divide rapidly under stimulation, giving rise to myocytes at various stages of maturation. ${ }^{3,10,22}$ Thus, the myocytes positive for cell cycle markers found in our patients would most likely constitute a population of amplifying myocytes that did not reach yet their terminally differentiated state and growth arrest.

On follow-up biopsies, in our study, the number of cycling myocytes increased significantly by $43 \%$ for $\mathrm{Ki}-67$ and $38 \%$ for MCM5 in responders and by 75 and $63 \%$ in nonresponders. This may be related to a stimulation of a healing process that becomes more pronounced in the presence of a more severe cell death and cardiac dysfunction. Nevertheless, the amount of proliferating cells in the myocardial tissue was lower than in other condition associated with persisting heart failure, ${ }^{3}$ and was higher in nonresponders than in responders suggesting that the contribution of this mechanism in our patient population was insufficient to explain itself the entire recovery of cardiac function. In addition, it raises the question of the possible use of systemic or cardiac growth factors (ie growth hormone, IGF-1, granulocyte colony-stimulating factor) and the enhancement of cardiac stem cell proliferation or recruitment for the treatment of inflammatory cardiomyopathy failing to respond to the conventional therapeutic regimens.

\section{Role of Cell Repair}

In this study, it is shown that myofibrillolysis is a constant, diffuse and consistent myocardiocyte damage in patients with active myocarditis and that it well correlates with the severity of LV dysfunction. Myofibrillolysis may occur through several mechanisms associated with myocardial inflammation including lysosomal and proteosomal protein degradation and caspase-3-mediated proteolysis. ${ }^{23}$

Indeed, activation of apoptotic pathways converges in the activation of caspase-3, a neutral, protease, which may degrade a large number of 
cytosolic proteins, including sarcomeric components. The morphometric analysis of pre and postrecovery from myocarditis left ventricular endomyocardial biopsies provides the first evidence that repair of myofibrillolysis may be a major mechanism of improvement of cardiac function, increasing cell myofibrillar content by an average value of $33 \%$. This figure attains to most of the myocardiocytes included in the histological sections and presumably to an extensive number of cells in the myocardium. In fact, it has been shown from biopsy and autopsy correlative studies that $\geq 5$ EMB samples, particularly if obtained from both ventricles are sufficiently representative of what occur in the entire myocardium. ${ }^{24}$

In addition, the myocytes appear bigger at recovery compared with baseline, probably as a consequence of a reactive hypertrophy to cell loss, being the extent of replacement fibrosis increased from 8 to $12 \%$. It derives that the increase of myofibrillar mass is a consistent contribute to the recovery of LV function and explains the linear correlation between percent increase of myofibrillar area with percent enhancement of LV ejection fraction. On the other hand, in the opposite situation of nonresponders, myofibrillar mass decreased by a mean value of $36 \%$ and correlated with percent reduction of left ventricular ejection fraction.

Further evidence of a strong activation of contractile protein synthesis comes in responding patients from molecular biology studies of $\alpha$ and $\beta$ isoforms of MHC at baseline and after recovery from myocarditis. In fact, both increased expression of $\alpha$-MHC and inhibition of $\beta$-MHC synthesis with enhanced $\alpha / \beta$ MHC ratio strongly suggest gene activation of foetal protein isoforms, ${ }^{25}$ that typically become operative in the process of cell repair.

\section{Limitation of the Study and Conclusions}

Our study suggests that recovery of cardiac function in patients with myocarditis responding to immunosuppression is associated with remarkable cellular events including strong inhibition of cell degeneration and death, activation of cell proliferation and mostly newly synthesized contractile proteins. Although the study is descriptive and no causeand-effect relationship is provided between cellular changes and the increase in LV contractility, the pronounced cell improvements observed in responders and the cell deterioration documented in nonresponders constitute a strong suggestion for this link and ascribe to them a main functional role.

\section{Acknowledgements}

The Ministry of University and Research projects 'Identification of signalling pathways involved in molecular repair and functional recovery of post-mitotic cells' (to MAR) and 'Myocarditis/
Inflammatory Cardiomyopathy: efficacy of a therapeutic approach tailored on patients' virologic and immunologic profile' (to AF) are acknowledged.

\section{References}

1 Wojnicz R, Nowalany-Kozielska E, Wojciechowska C, et al. Randomized, placebo-controlled study for immunosuppressive treatment of inflammatory dilated cardiomyopathy: two-year follow-up results. Circulation 2001;104:39-45.

2 Frustaci A, Chimenti C, Calabrese F, et al. Immunosuppressive therapy for active lymphocytic myocarditis. Virological and immunological profile of responders vs nonresponders. Circulation 2003;107: 857-863.

3 Chimenti C, Kajstura J, Torella D, et al. Senescence and death of primitive cells and myocytes lead to premature cardiac aging and heart failure. Circ Res 2003;93:604-613.

4 Aretz H, Billingham ME, Edwards WD, et al. Myocarditis: a histopathologic definition and classification. Am J Cardiovasc Pathol 1987;1:3-14.

5 Chimenti C, Calabrese F, Thiene G, et al. Inflammatory left ventricular microaneurysms as a cause of apparently idiopathic ventricular tachyarrhythmias. Circulation 2001;104:168-173.

6 Frustaci A, Perrone GA, Gentiloni N, et al. Reversible dilated cardiomyopathy due to growth hormone deficiency. Am J Clin Pathol 1992;97:503-511.

7 Di Marcotullio L, Ferretti E, De Smaele E, et al. REN(KCTD11) is a suppressor of Hedgehog signaling and is deleted in human medulloblastoma. Proc Natl Acad Sci USA 2004;101:10833-10838.

8 Frustaci A, Kajstura J, Chimenti C, et al. Myocardial cell death in human diabetes. Circ Res 2000;87:11231132.

9 Didenko VV, Tunstead JR, Hornsby PJ. Biotin-labeled hairpin oligonucleotides: probes to detect doublestrand breaks in DNA in apoptotic cells. Am J Pathol 1998;152:897-902.

10 Urbanek K, Quaini F, Tasca G, et al. Intense myocyte formation from cardiac stem cells in human cardiac hypertrophy. Proc Natl Acad Sci USA 2003;100: 10440-10445.

11 Tanaka T, Knapp D, Nasmyth K. Loading of a Mcm protein onto DNA replication origins is regulated by Cdc6p and CDKs. Cell 1997;90:649-660.

12 Scholzen T, Gerdes J. The Ki-67 protein: from the known and the unknown. J Cell Physiol 2000;182: 311-322.

13 Parrillo JE, Cunnion RE, Epstein SE, et al. A prospective, randomized, controlled trial of prednisone for dilated cardiomyopathy. N Engl J Med 1989;321: 1061-1068.

14 Mason JW, O’Connell JB, Herskowitz A, et al. A clinical trial of immunosuppressive therapy for myocarditis: the Myocarditis Treatment Trial Investigators. N Engl J Med 1995;333:269-275.

15 Guerra S, Leri A, Wang X, et al. Myocyte death in the failing human heart is gender dependent. Circ Res 1999;85:856-866.

16 Heusel JW, Wesselschmidt RL, Shresta S, et al. Cytotoxic lymphocytes require granzyme B for the rapid induction of DNA fragmentation and apoptosis in allogeneic target cells. Cell 1994;76:977-987. 
17 Rodella L, Rezzani R, Zauli G, et al. Apoptosis induced by NK cells is modulated by the NK-active cytokines IL-2 and IL-12. Int Immunol 1998;10:719-725.

18 Kim PK, Kwon YG, Chung HT, et al. Regulation of caspases by nitric oxide. Ann NY Acad Sci 2002;962:42-52.

19 Fiers W, Beyaert R, Declercq W, et al. More than one way to die: apoptosis, necrosis and reactive oxygen damage. Oncogene 1999;18:7719-7730.

20 Leri A, Claudio PP, Li Q, et al. Stretch-mediated release of angiotensin II induces myocyte apoptosis by activating p53 that enhances the local renin-angiotensin system and decreases the Bcl-2-to-Bax protein ratio in the cell. J Clin Invest 1998;101:1326-1342.

21 Saraste A, Arola A, Vuorinen T, et al. Cardiomyocyte apoptosis in experimental coxsackievirus B3 myocarditis. Cardiovasc Pathol 2003;12:255-262.
22 Beltrami AP, Barlucchi L, Torella D, et al. Adult cardiac stem cells are multipotent and support myocardial regeneration. Cell 2003;114:763-776.

23 Condorelli G, Roncarati R, Ross J, et al. Heart-targeted overexpression of caspase- 3 in mice increases infarct size and depresses cardiac function. Proc Natl Acad Sci USA 2001;98:9977-9982.

24 Baandrup U, Florio RA, Olsen EG. Do endomyocardial biopsies represent the morphology of the rest of the myocardium? A quantitative light microscopic study of single v. multiple biopsies with the King's bioptome. Eur Heart J 1982;3:171-178.

25 Reiser PJ, Portman MA, Ning XH, et al. Human cardiac myosin heavy chain isoforms in fetal and failing adult atria and ventricles. Am J Physiol Heart Circ Physiol 2001;280:H1814-H1820. 